\title{
A MODEL OF SOLAR FLARES
}

\author{
P. A. Sturrock \\ (Institute for Plasma Research, Stanford University, Stanford, Calif., U.S.A.)
}

\begin{abstract}
A BSTRACT
A model of solar flares is proposed in which the preflare state comprises a bipolar magnetic-field structure associated with a bipolar photospheric magnetic region. At low heights, the magnetic-field lines are closed but, at sufficiently great heights, the lines are drawn out into an open structure comprising a bipolar flux tube containing a 'neutral sheet' or 'sheet pinch'. Such a sheet pinch is probably related to a coronal streamer. The energy stored in the closed-field region is derived from photospheric motion whereas energy stored in the open-field region is derived from the non-thermal energy flux which heats the corona and drives the solar wind.

The flare itself is identified with reconnection of magnetic field by the tearing-mode resistive instability. If the thickness of the sheet pinch is determined by resistive diffusion and a growth time of the bipolar region of order 1 day, the transverse dimension will be about $10^{4} \mathrm{~cm}$. The rise time of the tearing-mode instability is then a few seconds, compatible with the characteristic time of Type-III radio bursts. One can understand that the time-scale of the reconnection process is of order $10^{2}-10^{3} \mathrm{sec}$ if reconnection proceeds by the Petscheck mechanism, with the modification that resistive diffusion is replaced by the more rapid Bohm diffusion.

The evolution of a flare, according to this model, appears to fit a number of the observational characteristics of flares.
\end{abstract}

It seems unlikely that it will ever be possible to deduce the mechanism of solar flares directly and unambiguously from observations. Progress in understanding this complex phenomenon can probably come only by a long process of trial and error. In attempting to understand such a phenomenon as it is explained to him by observers, a theorist will sketch an interpretation which may develop into a full-fledged theory. Such a theory would be subjected to two types of tests: that of internal physical and mathematical consistency, and that of agreement between components of the theory and observational facts. Since the development of a detailed theory of such a phenomenon could well use up many man-years of theoretical labor, it is sensible to first examine a theory in outline form. This is roughly what is meant by constructing a 'model' of the phenomenon. My purpose here is to present a model of solar flares and I hope to learn from your response the extent to which characteristics of this model fit the characteristics of the real phenomenon.

A solar flare is an explosion which, as far as we can tell, usually occurs spontaneously. This implies that the basic mechanism of a solar flare is some kind of instability. It has been agreed for some time that a solar flare is an electromagnetic process (Cowling, 1953), and this implies that the instability is a plasma instability. Furthermore, it is generally believed that the energy released in the flare is stored in magnetic

Kiepenheuer (ed.), Structure and Development of Solar Active Regions, 471-479. C. I.A.U. 
form (Gold and Hoyle, 1960). Since a flare seems to occur in the chromosphere and corona, and has no apparent influence upon the photosphere, the magnetic field of a preflare state can not be in its lowest-energy state, consistent with the boundary conditions of given normal magnetic field at the photosphere. This means that there must be currents in the preflare magnetic-field pattern. However, since the corona has a low gas density and pressure, one might imagine that the magnetic field must be virtually force-free, and Schmidt (1968), following a similar suggestion by Gold (1964) has proposed that the preflare energy is stored in a force-free magnetic field.

One objection to this proposal is that such a force-free field will be composed of closed magnetic-field lines. It is then hard to understand how electrons accelerated at the very beginning of a flare can travel almost radially outward and produce a Type-III radio burst (Wild et al., 1963). The idea proposed by Schmidt and Gold also traces the source of flare energy to movement of magnetic-field lines at the photosphere. But this aspect of the idea is hard to reconcile with the occurrence of homologous flares (Ellison, 1963) which in their idealized form repeat exactly, without significant change in the photospheric magnetic field. For these reasons, one is persuaded to look for an alternative method for storing magnetic energy and an alternative method for providing this energy.

It is at this point relevant to direct one's attention to the solar wind (Dessler, 1967), the existence of which insures that some fraction of the magnetic-field lines emerging from the photosphere have an open configuration. If we now consider a simple bipolar magnetic region and construct a field pattern which has both closed and open magnetic-field lines, we arrive at a pattern of the type shown in Figure 1. This pattern contains a Y-type neutral point (or, more correctly, a 'neutral line'). The configuration of the magnetic field in the neighborhood of the neutral point is quite sensitive to the

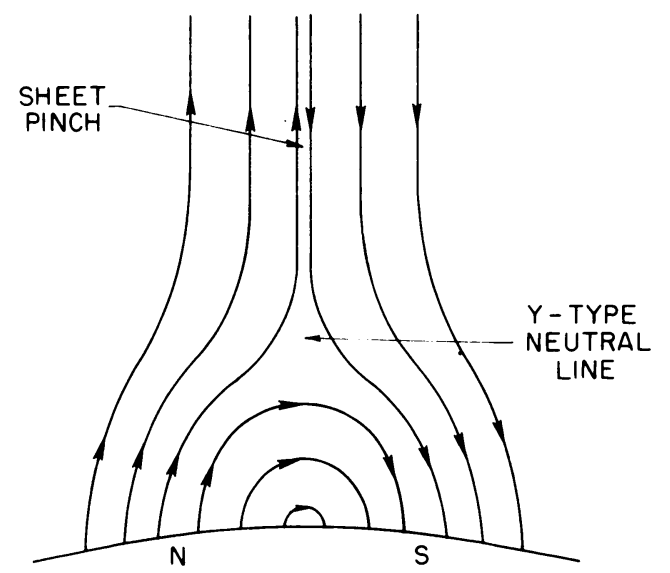

FIG. 1. Schematic representation of magnetic-field pattern above a bipolar magnetic region, showing the transition from closed field lines at low heights to open field lines at great heights, involving Y-type neutral line and sheet pinch. 
equality or inequality of gas pressures in the closed-field region and the open-field region. The diagram is drawn for the case that the gas pressure in the closed-field region is greater than that in the adjacent open-field region. The magnetic-field lines then take the form of a cusp, which is strongly reminiscent of the helmet shape often seen in coronal structures (Billings, 1966). If the gas pressure in the closed-field region were less than that in the adjacent open-field region, the field configuration in the neighborhood of the neutral point would be that of an inverted ' $T$ '. If there were a filament (quiescent prominence) in this structure, it would be comparatively low down above the line dividing regions of opposite magnetic polarity at the photosphere.

The important point about this configuration is the existence of a 'neutral sheet' or, as it is called in plasma physics, a 'sheet pinch' (Furth et al., 1963), in which there is a thin region of high-density plasma, the pressure of which balances the adjacent magnetic pressure. (Such a structure exists also in the magnetic tail of the earth (Ness, 1965).) A sheet pinch appears to be metastable, yet the associated magnetic field certainly contains 'free energy', which can be released if the magnetic field can be 'reconnected'. In the present model, such energy can be released without changing the connection of magnetic-field lines at the photosphere. The shape and location of the sheet pinch above a bipolar magnetic region are similar to that of a coronal streamer when a streamer is associated with a bipolar magnetic region. This suggests that a coronal streamer is in fact the visible manifestation of such a sheet pinch. Dr. Sheldon Smith of the NASA-Ames Research Laboratory has a very fine eclipse photograph which bears out this suggestion, and an article on this observation and interpretation will be published in the near future.

With this model of the preflare state we may now inquire into the development of the flare itself. Energy may be released by reconnection of the magnetic field, and such reconnection may be effected by the tearing-mode resistive instability analyzed in the linear approximation by Furth $e t a l$. (1963). The growth rate of this instability depends critically on the thickness of the sheet pinch, a dimension of which we have no direct knowledge. However, we can probably assign a lower limit to the thickness of the sheet pinch by considering the lifetime of the configuration and the relative diffusion rate of plasma and magnetic field. Spitzer (1962) gives a convenient formula for relating the diffusion time $\tau_{\mathrm{D}}$, the temperature $T$ of a fully ionized plasma, and the characteristic length scale $L$ :

$$
\tau_{\mathrm{D}} \approx 10^{-12 \cdot 7} T^{3 / 2} L^{2}
$$

If the temperature of the corona is taken to be $10^{6.4}{ }^{\circ} \mathrm{K}$, and if $\tau_{\mathrm{D}}$ is identified with the lifetime of a coronal streamer which is perhaps of order 1 day in an active region, i.e. $10^{5} \mathrm{sec}$, we find that $L$, which measures the thickness of the sheet pinch, is of order $10^{4} \mathrm{~cm}$, i.e. $0 \cdot 1 \mathrm{~km}$. This estimate of the thickness is much smaller than one might have supposed without considering the high conductivity and small diffusivity of the coronal plasma. 
The time-scale $\tau_{\mathrm{I}}$ of the most rapidly growing wave of the tearing-mode instability is given (Furth et al., 1963) by

$$
\tau_{\mathrm{I}}=\tau_{\mathrm{D}}^{\frac{1}{2}} \tau_{\mathbf{A}}^{\frac{1}{2}}
$$

where

$$
\tau_{\mathrm{A}}=L v_{\mathrm{A}}^{-1},
$$

$v_{\mathrm{A}}$ being the Alfvén velocity given by

$$
v_{\mathrm{A}}=B(4 \pi \rho)^{-\frac{1}{2}},
$$

where $B$ is the mean magnetic-field strength and $\rho$ the mean gas density. Since the magnetic pressure inside the sheet is being balanced by gas pressure on the outside, the Alfvén velocity will in fact be determined by the plasma temperature and be comparable with the speed of sound. For $T=10^{6.4}{ }^{\circ} \mathrm{K}$, we find that $v_{\mathrm{A}}=10^{7 \cdot 5} \mathrm{~cm} \mathrm{sec}^{-1}$. This means that, for the sheet we are considering, $\tau_{\mathrm{A}}=10^{-3 \cdot 5} \mathrm{sec}$, so that $\tau_{\mathrm{I}}=10^{\cdot 7} \mathrm{sec}$.

The above time-scale of $5 \mathrm{sec}$ for the growth rate of the tearing-mode instability may seem to be too short to be relevant to solar flares. However, this is probably not the case. Type-III radio bursts frequently occur at the beginning of a solar flare. The fine structure of a Type-III radio burst can have a time-scale of about $1 \mathrm{sec}$. Hence, the growth rate which we have computed is none too short. Indeed, we should possibly conclude that the growth rate should be even more rapid so that the thickness of the sheet pinch is typically even less than we have assumed.

The preceding considerations indicate that the onset of instability is very rapid, and further calculations of this stage of the flare process indicate that sufficient electrons will be accelerated to high enough energy to explain the principal characteristics of Type-III radio bursts. However, the main energy release of a flare must be effected by a process with very different parameters. The time-scale for release of the bulk of the energy of a very large flare (Smith and Smith, 1963) is of order $10^{3} \mathrm{sec}$. Since the transverse dimensions of a very large flare are typically $10^{10} \mathrm{~cm}$, this implies that the mean relative diffusion velocity of magnetic field and plasma is about $10^{7} \mathrm{~cm}$ $\mathrm{sec}^{-1}$. Petschek (1964) has considered the possibility that this very rapid reconnection takes place in a very thin layer (the magnetohydrodynamic treatment of his mechanism leads to a sheet thickness of order $10^{-4} \mathrm{~cm}$ ). However, the magnetohydrodynamic equations may not be applied to the coronal plasma when the relevant length-scale is so very small.

If the Type-III phenomenon represents the initial build-up of the tearing-mode instability, then the 'flash phase' of a flare presumably represents a process which takes place after the instability has developed to the highly non-linear level of amplitude at which the plasma is best described as 'turbulent'. If this is so, the rapid reconnection of the magnetic-field lines may be ascribed to anomalous diffusion in a turbulent plasma. In order to determine whether the reconnection rate can be sufficiently rapid, we follow Spitzer (1962) in adopting the Bohm diffusion coefficient 
as that which characterizes a highly turbulent plasma:

$$
D_{B}=\frac{1 c k T}{16 \frac{\mathrm{eB}}{\mathrm{e}}} \approx 10^{2 \cdot 6} \mathrm{~B}^{-1} T .
$$

This means that the relative diffusion velocity is given by

$$
v_{\mathrm{D}} \approx D b^{-1} \approx 10^{2 \cdot 6} B^{-1} b^{-1} T,
$$

where $b$ is the length-scale characterizing magnetic and plasma fluctuations transverse to the magnetic field. If $b$ is identified with the small-scale structure developed by the tearing-mode instability, we find from the article by Furth et al. (1963) that we should adopt

$$
b \approx\left(\tau_{\mathrm{D}} / \tau_{\mathrm{A}}\right)^{-\frac{1}{4}} L .
$$

For the particular values we adopted, this gives $b \approx 10^{2} \mathrm{~cm}$. Since we are considering the state resulting from the onset of instability, we need to evaluate the plasma temperature after the occurrence of instability rather than before it. This temperature may be estimated by equating the magnetic-energy density $(1 / 8 \pi) B^{2}$ before the flare to the plasma energy density $2 \times \frac{3}{2} n k T$ after the flare (where $n$ is the number density of electrons and of ions outside the initial sheet pinch). If we take $B \approx 10^{2}$ gauss and $n \approx 10^{10} \mathrm{~cm}^{-3}$, we find that the plasma is heated to a temperature of $10^{8}{ }^{\circ} \mathrm{K}$. When these values are substituted into Equation (6), we find the anomalous diffusion velocity to be $10^{6.6} \mathrm{~cm} \mathrm{sec}^{-1}$, which is very close to the required value of $10^{7} \mathrm{~cm} \mathrm{sec}^{-1}$ quoted above. It seems, therefore, that the very rapid rise-time of the light curve of a flare may be ascribed to anomalous diffusion, of the Bohm type, which follows the development of very small-scale turbulence resulting from the tearing-mode instability.

The above considerations may perhaps best be interpreted as a modification of the Petschek (1964) model of the reconnection of the magnetic-field lines in a solar flare, in which the resistive layer is replaced by a turbulent layer, and resistive diffusion by Bohm diffusion. The thickness of the layer is then estimated to be $10^{2} \mathrm{~cm}$ (which is in fact the transverse length-scale of the most rapidly growing wave of the tearingmode instability, in the model discussed earlier) rather than the $10^{-4} \mathrm{~cm}$, as estimated by Petschek.

The decay time of the light curve of a flare is typically about ten times the rise time (Smith and Smith, 1963), and this part of the curve has an exponential form. It appears, therefore, that the slow decay is likely to be caused by the slow diffusion of heat and energetic particles out of the instability region down to the chromosphere, where the $\mathrm{H} \alpha$ and other prominent emission lines are produced.

The gross characteristics of the evolution of a flare according to the above model is shown schematically in Figure 2. We see that the stream of heat and particles, as guided along magnetic-field lines to the chromosphere, will give rise to a pair of bright filaments, as is typically observed (Ellison, 1963). As the process continues, the two regions at which heating occurs will move progressively away from the line dividing 


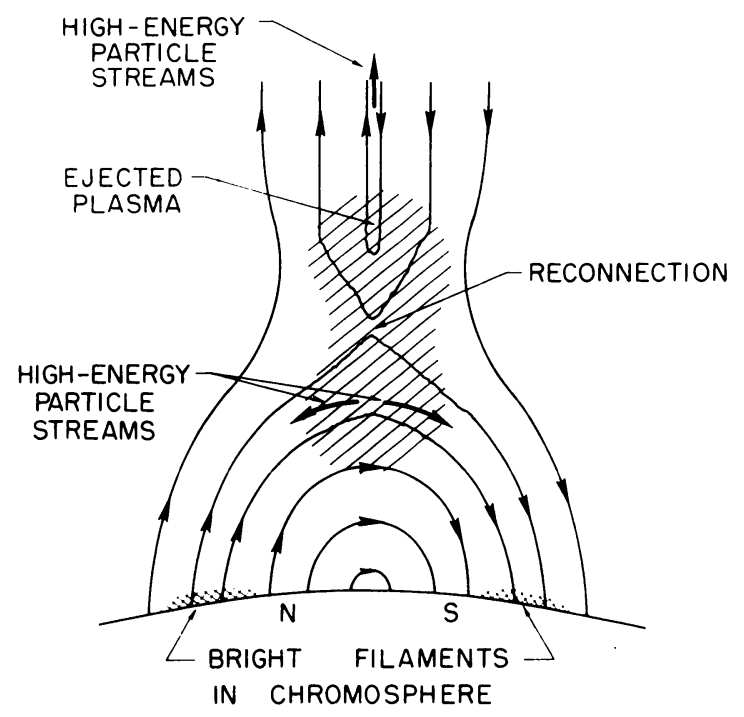

FIG. 2. Schematic representation of reconnection of magnetic-field lines during a flare, showing ejection of plasma and heating of chromosphere by particle streams to form two bright filaments.

opposite magnetic polarities, at the photosphere, giving the appearance that the two bright filaments are moving slowly away from each other. Since the plasma effected by the reconnection process is believed to be highly turbulent, there will be stochastic acceleration (Sturrock, 1966a; Hall and Sturrock, 1968) of both electrons and protons, but these will escape only slowly from the 'magnetic bag' formed by the disordered magnetic field. Such a moving cloud of electrons might give rise to what is called a moving Type-IV radio burst (Wild et al., 1963). Furthermore, the collisionless shock wave which probably forms ahead of the outward-moving plasma cloud may be the origin of a Type-II radio burst (Wild et al., 1963). One may also note that this magnetic cloud will ultimately be ejected completely from the Sun. On arrival in the vicinity of the Earth, this cloud would give rise to a geomagnetic storm.

The paper presented at this meeting by Rust (1968) ascribes surges and Ellerman bombs to small magnetic regions surrounded by larger regions of opposite magnetic polarity, such as 'satellite sunspots'. It appears that the flare process described in this article may be applied also to a ring-type sheet pinch, such as may form above the boundary of a satellite sunspot. If the Y-type neutral line of this configuration is low in the atmosphere, perhaps at chromospheric heights, the emission line may well be absorbed at the centre of the line, as is characteristic of the 'moustache' shape of the emission features of Ellerman bombs (Severny, 1964). The ejected plasma would then be much more dense than is typical of flares of the type we have discussed earlier, which lead to the ejection of coronal plasma. The ejection of a column of chromospheric plasma may well be the proper interpretation of surges. 
In conclusion, we shall inquire briefly into the origin of the energy which is stored in the open magnetic-field pattern shown in Figure 1. This is believed to be derived from the non-thermal energy flux which goes to heat the corona and drive the solar wind. This, according to Osterbrock (1961) is typically $10^{7} \mathrm{erg} \mathrm{cm}^{-2} \mathrm{sec}^{-1}$, but may be higher by a factor of 10 in active regions. Since the area covered by a large flare is $10^{20} \mathrm{~cm}^{2}$ and the recovery time between a series of intense homologous flares (Ellison, 1963) is about 1 day $\left(10^{5} \mathrm{sec}\right)$, we see that the total non-thermal energy ejected in this time, into this region, is $10^{33}$ erg. Hence we can explain the energy released during a flare if only a few percent of this energy is converted into the "free energy' associated with the open magnetic-field structure of Figure 1.

Since accounts of this model were first published (Sturrock, 1966b, 1968), it has come to the author's attention that very similar ideas have previously been expressed by Carmichael (1964).

\section{Acknowledgements}

This work was supported by the Air Force Office of Scientific Research, Office of Aerospace Research, United States Air Force, under Contract AF 49(638) 1321.

\section{References}

Billings, D.E. (1966) A Guide to the Solar Corona, Academic Press, New York, p. 65.

Carmichael, H. (1964) in Proc. AAS-NASA Symp. on the Physics of Solar Flares, NASA SP-50, National Aeronautics and Space Administration, Washington, D.C., p. 451.

Cowling, T.G. (1953) in The Sun, Ed. by G. P. Kuiper, University of Chicago Press, Chicago, p. 583.

Dessler, A.J. (1967) Rev. Geophys., 5, 1.

Ellison, M.A. (1963) Q.J.R. astr. Soc., 4, 62.

Furth, H.P., Killeen, J., Rosenbluth, M.N. (1963) Phys. Fluids, 6, 459.

Gold, T. (1964) in Proc. AAS-NASA Symp. on the Physics of Solar Flares, NASA SP-50, National Aeronautics and Space Administration, Washington, D.C., p. 389.

Gold, T., Hoyle, F. (1960) Mon. Not. R. astr. Soc., 120, 89.

Hall, D.E., Sturrock, P.A. (1968) Phys. Fluids 10, 2620.

Ness, N.F. (1965) J. Geophys. Res., 70, 2989.

Osterbrock, D.E. (1961) Astrophys. J., 134, 347.

Petschek, H.E. (1964) in Proc. AAS-NASA Symp. on the Physics of Solar Flares, NASA SP-50, National Aeronautics and Space Administration, Washington, D.C., p. 425.

Rust, D.M. (1968) in the present volume, p. 77.

Schmidt, H.U. (1968) in the present volume, p. 95.

Severny, A. B. (1964) A. Rev. Astr. Astrophys., 2, 363.

Smith, H.J., Smith, E. v. P. (1963) Solar Flares, MacMillan, New York, p. 102.

Spitzer, L. (1962) Physics of Fully Ionized Gases, Interscience, New York, 2nd ed., pp. $42,47$.

Sturrock, P.A. (1966a) Phys. Rev., 141, 186.

Sturrock, P.A. (1966b) Nature, 211, 695.

Sturrock, P.A. (1968) Solar Flares, in Proc. Enrico Fermi int. Summer School of Physics, 39th Course: Plasma Astrophysics, Varenna, Italy, 1966, p. 168.

Wild, J. P., Smerd, S. F., Weiss, A. A. (1963) A. Rev. Astr. Astrophys., 1, 305. 


\section{DISCUSSION}

De Jager: Any theory of flares should explain that the greater part of the energy of the larger types of flares is contained in particles accelerated to energies of the order of $10-100 \mathrm{KeV}$. In Sturrock's flare theory the optical flare is explained as caused by the collisions of chromospheric matter with downward-moving energetic particles. With a view to the fairly great energy which these particles presumably have it looks very difficult to produce anything like the optical flare by this interaction. The energy will mainly be emitted at shorter wavelength, not in $\mathrm{H} \alpha$ and other lines.

Sturrock: The primary energy-release mechanism is the tearing-mode instability which gives rise to strong electric fields associated with intense filamentary currents. When such an electric field develops in an open-field region in a low-density part of the corona, one expects that a large part of the energy released by the instability will appear as high-energy electrons and protons. However, the electric field associated with the closed-field region is developed at smaller heights and therefore in regions of higher density. The main result of the electric field is likely to be a conduction current rather than a runaway current, so that the resulting heating of the chromosphere is better interpreted as being due to thermal conduction from coronal heights, rather than a stream of high-energy particles.

De Feiter: An additional difficulty is that the downward motion of these energetic particles should last as long as the optical flare (hence $10 \mathrm{~min}$ to 1 or more hours). Since a plasma excited to high energies $(10-100 \mathrm{KeV})$ will lose its energy in a few minutes, Sturrock's theory makes necessary the assumption of a continuous acceleration of energetic particles, during a time as long as the period of visibility of the optical flare.

Sturrock: Suppose a large flare releases $10^{32} \mathrm{erg}$ in a closed-field region of height $10^{10} \mathrm{~cm}$ and area $10^{20} \mathrm{~cm}^{2}$. If this energy is radiated in $10^{3} \mathrm{sec}$, the downward heat flux will be $10^{9} \mathrm{erg} \mathrm{cm}^{-2} \mathrm{sec}^{-1}$, compatible with a maximum temperature of $10^{7.3}{ }^{\circ} \mathrm{K}$. To meet the total energy requirement, the mean density must be $10^{10} \mathrm{~cm}^{-3}$. The time-scale for cooling of this hot gas by bremsstrahlung radiation is in excess of $10^{5} \mathrm{sec}$, much longer than the time-scale for cooling by conduction. The timescale for cooling by gyro-radiation is about $10^{6} \mathrm{sec}$. Hence the present theory does not require acceleration after the flash phase of a flare.

Newkirk: Your model requires the existence of open magnetic-field lines above the active region. How do these lines become opened initially?

Sturrock: There have been open field lines as long as there has been a solar wind. The boundary between closed and open field lines is sensitive to the pressure differential between the plasma in these two regions. Since plasma in the closed field region cannot escape, it tends to have a higher temperature, density and pressure than that in the adjacent open field region. It seems that this allows a progressive ejection of closed field lines along the sheet pinch so that the bipolar flux tube grows from the inside out.

Wilcox: When energetic solar flare particles are detected by spacecraft near the Earth, does your model give any prediction for the configuration to be expected in the interplanetary magnetic field?

Sturrock: I have not investigated this question. My initial reaction is that the first high-energy particles would arrive along field lines with the normal spiral angle, but the main cloud of plasma would arrive with a magnetic field oriented transverse to the Sun-Earth line.

Davis: I have several questions on your interesting model; but most of them are related to the following feature. The tearing instability that produces the energy results in an increase in the flux of the arch region, i.e., the flux of the tubes both of whose ends pass into the photosphere. There is a corresponding decrease in the flux of the tubes that lead from the solar surface out into interplanetary space. After the flare is over and the energy supply is to be replenished, the original flux configuration must be restored. I do not understand how this can be done by heating the gas in the arches and pushing them up higher. Instead, it will be necessary to cut and reconnect lines of force.

Sturrock: The process which I just mentioned, in my reply to Dr. Newkirk, results in the ejection of closed field lines out along the sheet pinch into interplanetary space. As far as the flare region is concerned, these field lines are then effectively open. There is therefore a slow conversion of closed to open field lines without a bona fide reconnection by a resistive process.

Winckler: We note that the direct verification that Type-III bursts are actually caused by streams 
of relativistic electrons, by space measurements or X-ray bursts, is lacking at present. One should be cautious in using this idea to support a flare theory in a critical way.

Sturrock: There seems no doubt that Type-III bursts comprise electromagnetic radiation from a moving 'wake' of plasma oscillations in the corona. The only effective mechanism for exciting plasma oscillations is by an electron stream, and this supposition is supported by the high brightness temperatures of Type-III bursts which may be attributed to the two-stream instability. The additional fact that the radial velocity of the moving source is compatible with that of an electron stream results in a very strong case for the view that Type-III bursts are caused by electron streams. 